\title{
Beneficial Effects of a Semi-Intensive Stroke Unit are Beyond the Monitor
}

\author{
Carlo W. Cereda ${ }^{a, b}$ Paul M. George ${ }^{b}$ Lorenzo S. Pelloni ${ }^{a}$ \\ Paola Gandolfi-Decristophoris ${ }^{a}$ Michael Mlynash ${ }^{b} \quad$ Lucia Biancon Montaperto $^{a}$ \\ Costanzo Limoni ${ }^{a}$ Vesna Stojanova ${ }^{a}$ Roberto Malacrida ${ }^{c}$ Claudio Städler $^{a}$ \\ Claudio L. Bassettia, d

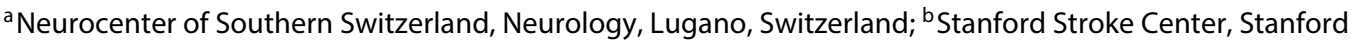 \\ University Medical Center, Stanford, Calif., USA; ' Intensive Care Unit, Ospedale Civico, Lugano, and \\ ${ }^{\mathrm{d}}$ Neurology Department, Inselspital Bern, Bern, Switzerland
}

\section{Key Words}

Acute management of stroke $\cdot$ Stroke unit $\cdot$ Intensive care unit $\cdot$ Mobile stroke system

\begin{abstract}
Background and Purpose: Precise mechanisms underlying the effectiveness of the stroke unit (SU) are not fully established. Studies that compare monitored stroke units (semiintensive type, SI-SU) versus an intensive care unit (ICU)based mobile stroke team (MST-ICU) are lacking. Although inequalities in access to stroke unit care are globally improving, acute stroke patients may be admitted to Intensive Care Units for monitoring and followed by a mobile stroke team in hospital's lacking an SU with continuous cardiovascular monitoring. We aimed at comparing the stroke outcome between SI-SU and MST-ICU and hypothesized that the benefits of SI-SU are driven by additional elements other than cardiovascular monitoring, which is equally offered in both care systems. Methods: In a single-center setting, we compared the unfavorable outcomes (dependency and mortality) at 3 months in consecutive patients with ischemic stroke or spontaneous intracerebral hemorrhage admitted to a stroke unit with semi-intensive monitoring (SI-SU) to a cohort of stroke patients hospitalized in an ICU and followed by a mo-
\end{abstract}

(๑) 2015 S. Karger AG, Basel

1015-9770/15/0392-0102\$39.50/0 bile stroke team (MST-ICU) during an equal observation period of 27 months. Secondary objectives included comparing mortality and the proportion of patients with excellent outcomes (modified Rankin Score (mRS) 0-1). Equal cardiovascular monitoring was offered in patients admitted in both SI-SU and MST-ICU. Results: 458 patients were treated in the SI-SU and compared to the MST-ICU $(n=370)$ cohort. The proportion of death and dependency after 3 months was significantly improved for patients in the SI-SU compared to MST-ICU ( $p<0.001 ; a O R=0.45 ; 95 \% \mathrm{Cl}: 0.31-0.65)$. The shift analysis of the $\mathrm{mRS}$ distribution showed significant shift to the lower mRS in the SI-SU group, $p<0.001$. The proportion of mortality in patients after 3 months also differed between the MST-ICU and the SI-SU $(p<0.05)$, but after adjusting for confounders this association was not significant $(\mathrm{aOR}=0.59$; $95 \% \mathrm{Cl}: 0.31-1.13)$. The proportion of patients with excellent outcome was higher in the SI-SU (59.4 vs. $44.9 \%, \mathrm{p}<0.001$ ) but the relationship was no more significant after adjustment $(\mathrm{aOR}=1.17 ; 95 \% \mathrm{Cl}: 0.87-1.5)$. Conclusions: Our study shows that moving from a stroke team in a monitored setting (ICU) to an organized stroke unit leads to a significant reduction in the 3 months unfavorable outcome in patients

C.S. and C.L.B. contributed equally to the manuscript.

\section{KARGER 125}

E-Mail karger@karger.com www.karger.com/ced
Dr. med. Carlo W. Cereda

Neurocenter of Southern Switzerland (EOC) Ospedale Civico

Via Tesserete 46, CH-6900 Lugano (Switzerland)

E-Mail carlo.cereda@eoc.ch 
with an acute ischemic or hemorrhagic stroke. Cardiovascular monitoring is indispensable, but benefits of a semi-intensive Stroke Unit are driven by additional elements beyond intensive cardiovascular monitoring. This observation supports the ongoing development of Stroke Centers for efficient stroke care.

(c) 2015 S. Karger AG, Basel

\section{Introduction}

The effectiveness of a dedicated stroke unit (SU) has been confirmed by large observational studies of routine clinical practice [1-3], and a geographically defined SU with a dedicated specialized team is associated with better clinical outcome than other therapeutic strategies like mobile stroke teams in non-monitored settings [4]. The precise mechanisms measured in terms of clinical outcome and mortality of the SU remains partially controversial and not fully established. The major benefits of a comprehensive acute stroke care are the monitoring of cardiovascular parameters, adherence to treatment protocols, training of the staff, presence of a dedicated stroke specialist, and early integration of neurorehabilitation programs [5-9].

Although inequalities in access to stroke unit care are globally improving [10], acute stroke patients may be admitted to Intensive Care Units (ICU) for monitoring and followed by a mobile stroke team in hospitals lacking an SU with continuous cardiovascular monitoring. Comparisons of care between a dedicated SU (with or without cardiovascular monitoring) and in ward departments (general medicine or general medicine with rehabilitation) are abundant $[11,12]$. Studies that compare monitored SUs (semi-intensive type) versus non-monitored structures are rare $[7,9]$, and a comparison between a geographically monitored SU (semi-intensive type, SISU) and a monitored (ICU-based) mobile Stroke Team system (MST-ICU) is lacking. We hypothesized that the exhaustive cardiovascular monitoring, as offered by an MST-ICU management approach, is not sufficient to explain the benefit of specialized stroke care. We report here the experience of a single hospital before and after the creation of a semi-intensive SU.

\section{Methods}

The study is designed with a 'before-after' approach to observe the functional outcomes, in terms of dependence (defined as modified Rankin Scale (mRS) of 3-5) and 3-month mortality (mRS of
6), in consecutive patients diagnosed with ischemic stroke or spontaneous intracerebral hemorrhage admitted to an SI-SU with dedicated specialized staff over 27 months. These results were compared with a retrospective cohort of patients hospitalized for stroke monitoring in the MST-ICU setting. The functional outcomes were pre-specified prior to the start of the study. All patients in the study were entered prospectively in a specified database (RADIAL,

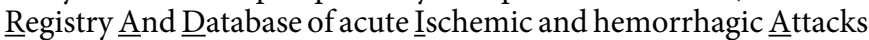
in Lugano). The web-based database was created before the beginning of the study. The study's main objective was to determine if the proportion of patients with unfavorable outcome (defined as 3 months mRS Scale 3-5 or mortality) differed between the period before and the period after the onset of the SI-SU. Secondary objectives included comparing mortality and the proportion of patients with excellent outcomes (mRS 0-1). Explorative analyses were carried out to assess potential predictors of unfavorable outcome. Additional, explorative subgroup analyses were performed with respect to the effects of the SI-SU on dependence and mortality after 3 months.

\section{Characterization of Treatment Concepts}

All principal treatment processes attributed to the two different stroke treatment concepts are summarized in table 1. Cardiovascular monitoring was equally offered in both SI-SU and MSTICU.

\section{Semi-Intensive Stroke Unit (SI-SU)}

The SI-SU is a 6-monitored bed unit located in the Neurocenter of Southern Switzerland, in the Ospedale Regionale di Lugano, Switzerland that is a certified Comprehensive Stroke Center, consistent with the EUSI/ESO [11, 13] and Swiss Stroke Society [14] criteria. In the SI-SU, the responsible medical team is represented by stroke neurologists (with National Certification for Cerebrovascular Diseases; http://www.sgkn.ch), dedicated team nurses with stroke experience, internists and a multidisciplinary neurorehabilitation team (for details see table 1). Patients were monitored in the unit for a minimum of $24 \mathrm{~h}$. Patients, according to clinical evolution, were subsequently admitted to the adjacent neurology ward under the care of the same previously described team.

\section{Mobile Stroke Team with Monitored Stroke Beds in the}

Intensive Care Unit (MST-ICU)

In this care concept, the stroke patient is treated by a multidisciplinary care team in the ICU and by a mobile stroke team represented by stroke neurologists (with National Certification for Cerebrovascular Diseases; http://www.sgkn.ch), serving as consultants. Additionally, a mobile multidisciplinary neurorehabilitation team followed these patients, for details see table 1 . This unit also has access to neurosurgeons, neuroradiologists, and vascular surgeons present in the hospital. Patients were monitored in the unit for a minimum of $24 \mathrm{~h}$. After the ICU, patients were transferred to the departments of Neurology (approx. 90\%) or Internal Medicine $(10 \%)$ with neurology following as a consulting team.

\section{Patient Selection}

Patients included those admitted to the SI-SU or MST-ICU with a diagnosis of acute cerebrovascular ischemic stroke (AIS) or spontaneous intracerebral hemorrhage (ICH). Informed written consent of patients or of their relatives was required. Criteria for inclusion were clinical and/or radiologic diagnosis of AIS or ICH, 
Table 1. Stroke treatment processes definition

\begin{tabular}{lll}
\hline Intervention & SI-SU & MST-ICU \\
\hline Beds (medical responsible) & Stroke unit (neurology dept.) & Intensive care (ICU dept.) \\
Cardiovascular monitoring & Blood pressure, heart rhythm, blood sugar, & Blood pressure, heart rhythm, blood sugar, \\
& oxygen saturation, fluid balance & GCS, NIHSS, and ad hoc \\
Neurological monitoring & GCS, NIHSS, and ad hoc & Mobile unit (consultant/on call) \\
Vascular neurology team & On site & On site \\
Internal medicine consultants & On call & On call \\
Cardiology consultants & On call & In house, on call \\
Neuroradiology & In house, on call & In house, on call \\
Neurosurgery & In house, on call & In house, on call \\
Neurosonology & On site, on call & Present \\
Stroke guidelines/treatment pathways & Present & On call \\
Acute neuro-rehabilitation program & On site and on call & On call \\
Speech and language therapists & On site and on call & On call \\
Neuro-rehabilitation team visit & Twice a week and on call & Absent \\
Stroke-rehabilitation team meeting & Once a week & On call \\
Neuropsicology consulting & On site & Less frequent $(<10 /$ year) \\
Stroke training/update conferences & Frequent (>10/year) & Partially present \\
Stroke focused nursing program & Present & Present \\
Stroke research & Present & \\
\hline
\end{tabular}

hospitalization occurred within the first $24 \mathrm{~h}$ of onset of symptoms, and age $>18$. Exclusion criteria were patients already institutionalized or severely disabled (with documented mRS $>3$ ) before the AIS or ICH, preexisting dementia, severe comorbidity (life expectancy $<12$ months) or in which an early decision (within $48 \mathrm{~h}$ ) of care withdrawn is made (non-maximalist therapy, neurological team always involved in the decision). Patients with subarachnoid hemorrhage or other secondary hemorrhage (e.g. arteriovenous malformation) as well as patients with TIA were also excluded from analysis. The selection criteria of subjects recruited during the before period (MST-ICU) were identical to those of the period after (SI-SU).

\section{Data Collection}

The data was collected by the treating neurology team in the acute phase of the stroke (first 7 days) and completed with a followup at 3 months. The monitoring during the interim ( 3 months) was done through independent clinical assessment or telephone survey by trained and certified personnel. The data thus collected were included in the prospective database. The following data were recorded: sociodemographic characteristics (age, gender), vascular risk factors, stroke severity (by NIHSS), degree of disability according to the mRS $[15,16]$ before stroke onset and clinical stroke classification according to the Trial of Org 10172 in Acute Stroke Treatment (TOAST) protocol $[17,18]$.

\section{Ethics, Role of the Funding Source}

The local Ethics Committee examined and approved the study protocol (CE number 2278). The sponsor of the study (EOC) had no role in study design, data collection, data analysis, data interpretation, or writing of the report. All patients gave informed consent to participate in the study and be registered in the RADIAL database.

\section{Statistical Analysis}

The sample size was calculated based on the expected primary endpoints of mortality and dependency at three months between the two cohorts. In particular, an absolute reduction of $10 \%$ of the proportions of unfavorable outcome was expected to be clinically relevant. Based on these assumptions, 350 patients per group were required to achieve a power of $80 \%$ with a significance level of $5 \%$ $\left(\chi^{2}\right.$ test). We created dichotomous variables for the outcomes after three months: death and dependency (unfavorable outcome, defined as mRS 3-6), mortality defined as $m R S=6$, and excellent outcome defined as $\mathrm{mRS} \leq 1$. We also performed a shift analysis of the mRS, according to a recent publication [19]. In particular, we compared the distribution of the mRS between the groups using Cochran-Mantel-Haenszel (CMH) statistics with the computation option of the modified RIDIT scores, which represent the expected values of the order statistics for the uniform distribution on $(0,1)$. Means are given with standard deviations $( \pm S D)$. The association between categorical variables was investigated using the mean of chi-squared test $\left(\chi^{2}\right)$. Numerical variables were compared using the mean of the Student's t test and when applicable using the Mann-Whitney U Test. All tests were two-tailed and considered significant if $\mathrm{p}<0.05$. Univariable logistic regression models were used to determine the effect of hospitalization in the SU on dependency and mortality and on mortality at 3 months by calculating the odds ratio (OR) and their relative 95\% confidence intervals (95\% CI).

A multivariable logistic regression model was used to explore the possible risk factors related to the dependency and mortality after 3 months. The results were adjusted for age, sex, and hospitalization in SI-SU. Based on the outcome of this analysis, subsequent models used to determine the effect of the hospitalization in the SI-SU on outcome at 3 months were adjusted for age, sex, NIHSS on admission, type of stroke, and diabetes. 


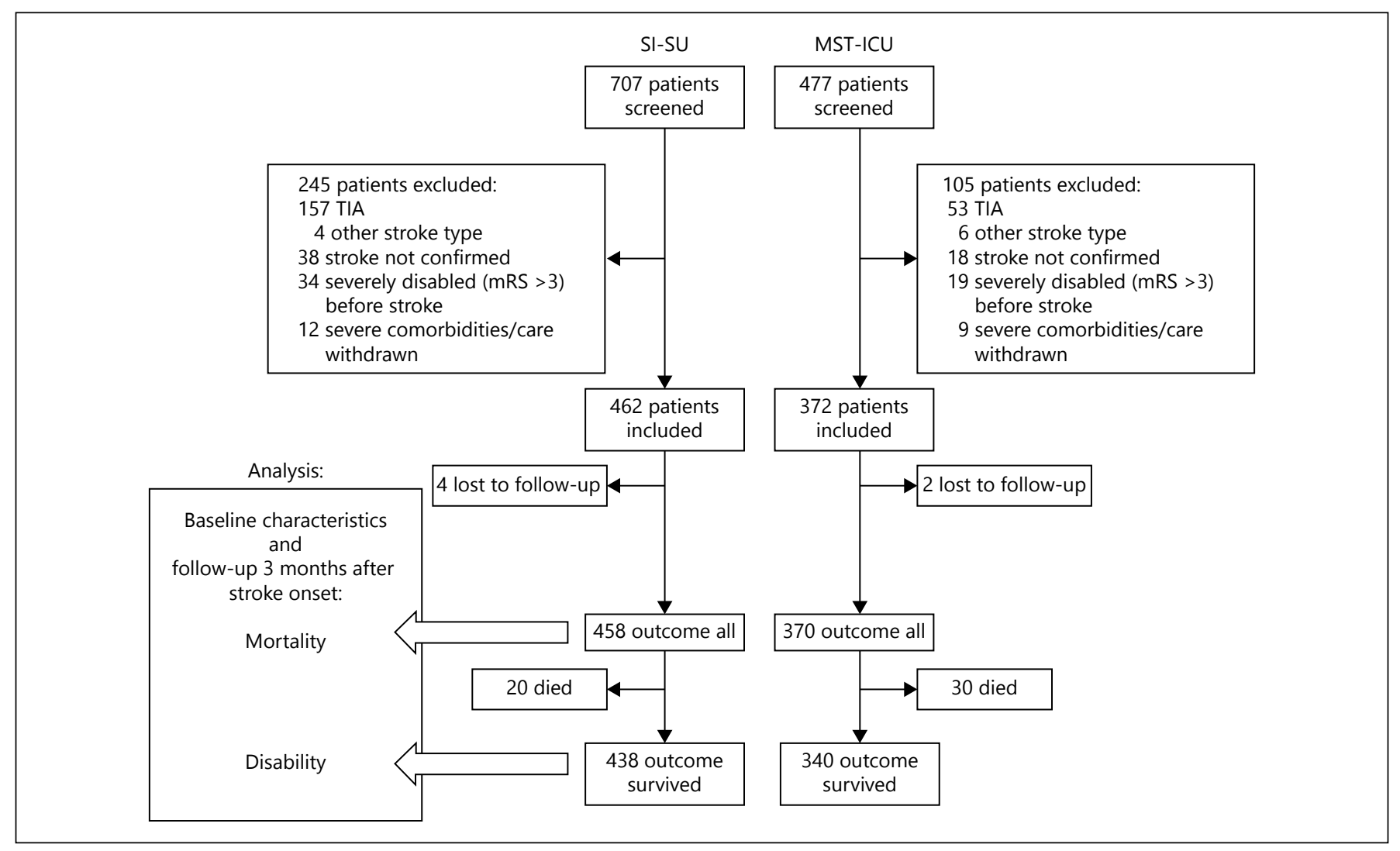

Fig. 1. Patient participation flow chart.

Explorative subgroup analysis models were made for age, gender, stroke severity on admission (NIHSS), and type of stroke (ischemic or hemorrhagic). A sensitivity analysis on dependency and death was performed which included patients who were previously excluded because of severe comorbidities and care withdrawn. All statistical analyses were performed in SPSS (IBM) 20.0 for Windows.

\section{Results}

For the MST-ICU period, patients were enrolled from 2008 to 2010. In 27 months a total of 477 patients were admitted. For the SI-SU period patients were enrolled from 2010 (start of the SI-SU phase, with no transition phase) until 2012. In 27 months a total of 707 patients were admitted. Overall, 350 patients did not fulfill the inclusion criteria for enrollment into the study. Causes for exclusion are listed in figure 1. A total of 828 patients where thus included in the analyses, 370 admitted to the MST-ICU and 458 to the SI-SU. In table 2, we reported baseline characteristics for the two groups (before, MSTICU and after, SI-SU) included in the study. Multivari- able exploratory analysis of possible predictors of unfavorable outcome showed that NIHSS $>5$ (NIHSS 5-15, $\mathrm{OR}=3.48 ; 95 \%$ CI: $2.36-5.12$ and NIHSS $>15$, OR $=29.8$; 95\% CI: 16.17-54.89), hemorrhagic stroke $(\mathrm{OR}=3.61$; 95\% CI: 2.02-6.45), and diabetes (OR $=1.63$; 95\% CI: 1.08-2.5) were significant factors increasing the risk of unfavorable outcome.

The rate of unfavorable outcome (dependency and death) in patients after 3 months differed between the MST-ICU and the SI-SU ( $p<0.001$; table 3$)$, with a crude odds ratio (OR) of 0.45 (95\% CI: 0.33-0.61), indicating a decreased proportion of patients with unfavorable outcomes associated with a hospitalization in the SI-SU. After adjusting for age, sex, NIHSS on admission, type of stroke and diabetes this association remained significant $(\mathrm{aOR}=0.45 ; 95 \% \mathrm{CI}: 0.31-0.65)$. The rate of mortality in patients after 3 months differed between the MST-ICU and the SI-SU (table 3), with a crude odds ratio (OR) of 0.51 (95\% CI: 0.28-0.92), indicating a decreased proportion of mortality in patients associated with a hospitalization in the SI-SU. After adjusting for age, sex, NIHSS on admission, type of stroke and diabetes, however, this as- 
Table 2. Baseline characteristics of studied population

\begin{tabular}{|c|c|c|c|c|}
\hline & \multicolumn{2}{|c|}{ SI-SU (n = 457 or 458$)$} & \multicolumn{2}{|c|}{ MST-ICU ( $\mathrm{n}=368$ or 370$)$} \\
\hline & $\mathrm{n} /$ total & $\%$ & $\mathrm{n} /$ total & $\%$ \\
\hline Female & $196 / 458$ & 42.8 & $142 / 370$ & 38.4 \\
\hline Age, mean $( \pm S D)$ & 70.7 & $(12.8)$ & 70.1 & $(14.6)$ \\
\hline $18-55$ & $62 / 457$ & 13.6 & $60 / 368$ & 16.3 \\
\hline $56-74$ & $192 / 457$ & 42.0 & $136 / 368$ & 37.0 \\
\hline $75-84$ & $141 / 457$ & 30.9 & $124 / 368$ & 33.7 \\
\hline$\geq 85$ & $62 / 457$ & 13.6 & $48 / 368$ & 13.0 \\
\hline \multicolumn{5}{|l|}{ NIHSS on admission } \\
\hline $0-4$ & $263 / 458$ & 57.4 & $193 / 370$ & 52.2 \\
\hline $5-15$ & $143 / 458$ & 31.2 & $114 / 370$ & 30.8 \\
\hline$>15$ & $52 / 458$ & 11.4 & $63 / 370$ & 17.0 \\
\hline \multicolumn{5}{|l|}{ Stroke type } \\
\hline Ischemic & $420 / 458$ & 91.7 & $324 / 370$ & 87.6 \\
\hline Hemorrhagic & $38 / 458$ & 8.3 & $46 / 370$ & 12.4 \\
\hline \multicolumn{5}{|l|}{ TOAST classification } \\
\hline Macroangiopathy & $64 / 458$ & 14.0 & $67 / 370$ & 18.1 \\
\hline Microangiopathy & $106 / 458$ & 23.1 & $89 / 370$ & 24.1 \\
\hline Cardioembolic & $144 / 458$ & 31.4 & $128 / 370$ & 34.6 \\
\hline Other & $47 / 458$ & 10.3 & $49 / 370$ & 13.2 \\
\hline Unknown & $97 / 458$ & 21.2 & $37 / 370$ & $10.0^{*}$ \\
\hline \multicolumn{5}{|l|}{ Comorbidities/risk factors } \\
\hline Diabetes mellitus & $86 / 458$ & 18.8 & $93 / 370$ & $25.1^{*}$ \\
\hline Atrial fibrillation & $84 / 458$ & 18.3 & $91 / 370$ & $24.6^{*}$ \\
\hline Current smoking & $125 / 458$ & 27.3 & $99 / 370$ & 26.8 \\
\hline Previous TIA/stroke & $68 / 458$ & 14.8 & $52 / 370$ & 14.1 \\
\hline Hypertension & $340 / 458$ & 74.2 & $267 / 370$ & 72.2 \\
\hline Dyslipidemia & $310 / 458$ & 67.7 & $204 / 370$ & $55.1^{* *}$ \\
\hline
\end{tabular}

Table 3. Clinical outcome (modified Rankin Scale, mRS) after 3 months follow-up with OR for SI-SU treatment and proportions of stroke patients

\begin{tabular}{|c|c|c|c|c|c|c|}
\hline & \multirow[t]{2}{*}{ OR $(95 \% \mathrm{CI})$} & \multirow[t]{2}{*}{$\mathrm{aOR}(95 \% \mathrm{CI})$} & \multicolumn{2}{|c|}{$\begin{array}{l}\text { SI-SU } \\
(\mathrm{n}=458)\end{array}$} & \multicolumn{2}{|c|}{$\begin{array}{l}\text { MST-ICU } \\
(\mathrm{n}=370)\end{array}$} \\
\hline & & & $\mathrm{n} /$ total & $\%$ & $\mathrm{n} /$ total & $\%$ \\
\hline Unfavorable outcome (mRS 3-6) & $0.45(0.33-0.61)$ & $0.45(0.31-0.65)$ & $113 / 458$ & $24.7^{* *}$ & $155 / 370$ & 41.9 \\
\hline Mortality $(m R S=6)$ & $0.51(0.28-0.92)$ & $0.59(0.31-1.13)$ & $20 / 458$ & $4.4^{*}$ & $30 / 370$ & 8.1 \\
\hline Excellent outcome (mRS 0-1) & $0.99(0.89-1.09)$ & $1.17(0.87-1.5)$ & $272 / 458$ & $59.4^{* *}$ & $166 / 370$ & 44.9 \\
\hline mRS at 3 months, mean $( \pm S D)$ & & & \multicolumn{2}{|c|}{$1.6[1.6]^{* *}$} & \multicolumn{2}{|c|}{$2.2(1.9)$} \\
\hline
\end{tabular}

sociation was no longer significant $(\mathrm{aOR}=0.59 ; 95 \% \mathrm{CI}$ : $0.31-1.13$ ). Patients in the SI-SU period showed a higher proportion of excellent outcomes at three months followup when compared to patients enrolled in the MST-ICU (table 3). Figure 2 shows the details of the mRS propor- tions at three months for the two groups of patients. The shift analysis of the mRS distribution showed significant shift to the lower $m R S$ in the SI-SU group, $p<0.001$. The Hodges-Lehman median difference in the scores was 1 (95\% CI: 0-1). A sensitivity analysis, also including the 
Fig. 2. Distribution of $\mathrm{mRS}$ at 3 months according to different treatment concept allocations. Comparison of the mRS distribution showed significant shift to the lower $\mathrm{mRS}$ in favor of the SI-SU group, $\mathrm{p}<0.001$.

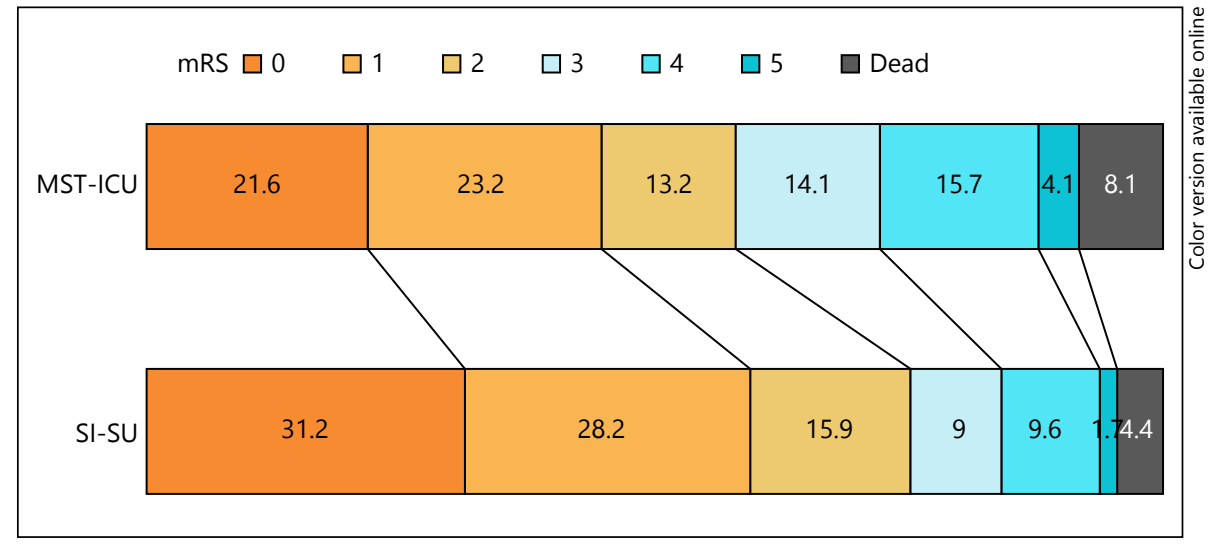

patients excluded for severe comorbidities/care withdrawn, confirmed the association between admission in the SI-SU and a decreased rate of patients with unfavorable outcomes (OR $=0.47$; 95\% CI: 0.35-0.63).

Multivariable analyses of subgroups, used to determine the effects of the hospitalization in the SU on dependency and death (online suppl. fig. 3; www.karger.com/ doi/10.1159/000369919) showed that all subgroups of patients analyzed benefited from being hospitalized in the SI-SU. Similarly, we found that hospitalization in the SISU was associated with reduced mortality, even if this effect was not significant (online suppl. fig. 3). In the MST-ICU period (27 months), 22 (5.9\%) patients received thrombolysis therapy (3 intra-arterial, 19 intravenous), which corresponds to a rate of 0.81 lysis per month. In the SI-SU period (27 months), this rate is increased to 2 lysis per month, with a total of 63 thrombolysis ( $14 \%$ of patients, 8 intra-arterial, 55 intravenous lyses) registered over 27 months $(\mathrm{p}<0.001)$. The number of patients transferred from outside hospitals (included in the same care system EOC) also increased from 38/364 (10.4\%) versus $87 / 449(19.4 \%, \mathrm{p}<0.001)$.

\section{Discussion}

We have shown that moving from a stroke team in a monitored setting (MST-ICU) to an organized Stroke Unit (SI-SU) leads to a significant reduction in the 3 months unfavorable outcomes in patients with an acute ischemic or hemorrhagic stroke.

Assuming that the cardiovascular monitoring algorithm is offered equally in both SI-SU and MST-ICU, the study supports the hypothesis that the benefits of specialized SUs are explained by elements other than intense cardiovascular monitoring. Given the current stroke care environment, a randomized trial to prove the effectiveness of the SI-SU, although theoretically needed, would likely be unethical. In this context, we attempted to better understand the effectiveness of an organized comprehensive stroke center in a real-world setting (of high developed medical countries) in which acute stroke patients may be hospitalized in an ICU for monitoring if a monitored Stroke Unit is not available. Monitoring of physiological parameters in the acute phase of ischemic stroke is an important management tool that can improve significantly the quality of care provided: this monitoring allows easier detection and correction of complications, consequently having a favorable effect on outcome. Because of this, monitoring has been established as a fundamental component of SU care and benefits [9,20]. Although the importance of monitoring is not debated, this study tells that the effects of the SI-SU are likely to be driven by additional elements, which are missing in an MST-ICU management. The main differences between SI-SU and MST-ICU (see table 1) are (1) the primary medical responsibility (neurologist in SI-SU and intensive care physicians in MST-ICU; (2) the presence of an on-site vascular neurology team in SI-SU (versus a consulting role in MST-ICU); (3) a more developed acute neuro-rehabilitation program in SI-SU with regular neuro-rehabilitation team visits on site (2/week) and a regular stroke team and rehabilitation meeting (1/week); and (4) in SI-SU the stroke training/update conferences are more frequent (>10/year) along with stroke-focused nursing programs. As a consequence, likely factors in the SI-SU are a continuity of the primary neurology team and a harmonized neuro-rehabilitation program. A structured acute neurorehabilitation program with regular neuro-rehabilitation team visits, on-site speech and language therapists, regu- 
lar multidisciplinary stroke-rehabilitation team meetings and personalized neuropsychology consulting contribute to more cohesive care. This may be influenced by more training/updates on stroke, a stroke-focused nursing program, and active stroke research. In our study, the augmented numbers of thrombolysis and transfers from regional hospitals seemed to be indicative of this trend. We can speculate that the creation of an SI-SU is associated with a 'new perception and culture of stroke care'.

Our data also demonstrated a nonsignificant reduction of mortality in the SI-SU group. A previous nonrandomized study showed that a semi-intensive monitored SU when compared to a non-monitored conventional SU is efficacious in terms of mortality [21] with a reduction from 27.0 to $15.6 \%$. The rate of 3 months mortality in our population was substantially low (reduction to 4.4 from 8.1 than the Cochrane Review's rate or other previous reports) $[7,12,21-24]$. We believe that the highly intensive monitored caring in both treatment concepts may be a major reason for our low in-hospital mortality. For this reason it may be easier to prove superiority by comparing an SI-SU system to conventional non-monitored ward care. The absence of significant difference of the SI-SU system on mortality is probably driven by the low number of deaths in the two groups and the fact that the study was not powered for a comparison on mortality alone.

We were concerned that the difference of stroke severity, although nonsignificant, could be a confounding variable. Explorative analyses on predictors for unfavorable confirmed the importance of taking into account the effects of NIHSS on admission, type of stroke, and diabetes. Given this, we adjusted our multivariate analysis model according to these assumptions. Additionally, subgroup analyses revealed that all investigated groups of patients with respect to age, NIHSS, and stroke type were associated with a better outcome if hospitalized in the SI-SU, even if it was not significant. This lack of significance is probably explained by the fact that the study was not powered for this analysis and because of the relatively low number of patients in each sub-group.

We concede that our study has several limitations. The design allows for a degree of selection bias. To minimize the bias selection, we excluded patients who did not fulfil the admission criteria. As a consequence (that we consider marginal in our study), only those patients allocated in the ICU or SI-SU based on bed availability were included in the analysis. Excluding patients with a rapid decision to abstain from aggressive treatment may have varied in the two different periods, because of 2 different teams directly responsible for patient care. Nevertheless, we performed a sensitivity analysis including these patients, which confirmed the main results. As previously discussed, the present results must also be cautiously interpreted, since this is not a randomized study. We also acknowledge that theoretically the comparison of a treatment in two consecutive periods may be associated with a better outcome in the most recent period because of optimization of care, but we think that this issue is in our study less plausible because the two time frames are in close proximity to one another and because of the high standard of care in the two settings. In addition, the results of the ECASS III study were associated immediately (September 2008) with a change in practice (IV thrombolysis allowed up to $4.5 \mathrm{~h}$ ), already in the MST-ICU period. The amount of tPA in the 3-4.5 h time window did not differ between the SI-SU and MST-ICU periods ( $11 \%$ $(2 / 19)$ and $14 \%(8 / 55)$, respectively; $\mathrm{p}=0.658)$.

While our study concentrated on the clinical outcomes between two models of care, other topics such as economical and cost-effectiveness between care systems are also important for optimizing patient care. These assessments reside outside the scope of this study. In our case, the decision to create and recognize at federal level stroke centers and stroke units was made by the conference of the directors of the Health Ministers (GDK) in 2011 and led to the definition of certification criteria and procedures by the Swiss Federation of Clinical Neurosocieties (SFCNS) [14]. Nevertheless, national health reimbursement strategies that valorize specialized stroke care in Stroke Centers deserve further study.

\section{Summary}

Our single-center study shows that moving from a stroke team in a monitored setting (ICU) to an organized stroke unit with semi-intensive care monitoring (SI-SU) leads to a significant reduction of unfavorable outcomes in patients with an acute ischemic or hemorrhagic stroke. Cardiovascular monitoring is indispensable, but we think that the benefits are driven by additional elements offered by neurological and multidisciplinary competent team synergy, focused in a circumscribed setting (Stroke Unit or Center). This 'stroke-focused' approach offers better stroke management by continuity of the primary team, a multidisciplinary neuro-rehabilitation program, and comprehensive medical, nursing protocols, and treatment algorithms. Nevertheless, not all the beneficial mechanisms of an SI-SU concept are fully understood and future detailed studies addressing this issue are need- 
ed. Meanwhile, our study supports the creation and promotion of Stroke Centers (or Units) for contemporary and efficient stroke care.

\section{Acknowledgments}

Valeria Pifferini, BS and Jane Frangi, BS.

\section{Source of Funding}

ABREOC - Ente Ospedaliero Cantonale (EOC).

\section{Disclosure Statement}

All authors report no disclosure.

\section{Authors' Contributions}

C.W.C., C.S., C.L.B.: study design; data collection, analysis and interpretation; manuscript draft; critical revisions. L.S.P., V.S., L.B.M.: data collection, analysis and interpretation; manuscript draft. P.G.-D., M.M.: statistical analysis and interpretation; manuscript draft; critical revisions. P.M.G., R.M.: manuscript draft; critical revisions.

\section{References}

1 Donnan GA, Fisher M, Macleod M, Davis SM: Stroke. Lancet 2008;371:1612-1623.

2 Seenan P, Long M, Langhorne P: Stroke units in their natural habitat: systematic review of observational studies. Stroke 2007;38:1886-1892.

-3 Candelise L, Gattinoni M, Bersano A, Micieli G, Sterzi R, Morabito A: Stroke-unit care for acute stroke patients: an observational followup study. Lancet 2007;369:299-305.

4 Langhorne P, Dey P, Woodman M, Kalra L, Wood-Dauphinee S, Patel N, et al: Is stroke unit care portable? A systematic review of the clinical trials. Age Ageing 2005;34:324-330.

5 Douglas VC, Tong DC, Gillum LA, Zhao S, Brass LM, Dostal J, et al: Do the Brain Attack Coalition's criteria for stroke centers improve care for ischemic stroke? Neurology 2005;64: 422-427.

-6 Steiner T: Stroke unit design: intensive monitoring should be a routine procedure. Stroke 2004;35:1018-1019.

7 Walter A, Seidel G, Thie A, Raspe H: Semiintensive stroke unit versus conventional care in acute ischemic stroke or TIA - a prospective study in Germany. J Neurol Sci 2009;287: 131-137.

8 Sulter G, Elting JW, Langedijk M, Maurits NM, De Keyser J: Admitting acute ischemic stroke patients to a stroke care monitoring unit versus a conventional stroke unit: a randomized pilot study. Stroke 2003;34:101-104.

-9 Cavallini A, Micieli G, Marcheselli S, Quaglini $S$ : Role of monitoring in management of acute ischemic stroke patients. Stroke 2003;34: 2599-2603.
10 Glader EL, Edlund H, Sukhova M, Asplund K, Norrving B, Eriksson M: Reduced inequality in access to stroke unit care over time: a 15year follow-up of socioeconomic disparities in Sweden. Cerebrovasc Dis 2013;36:407411.

11 Ringelstein EB, Chamorro A, Kaste M, Langhorne P, Leys D, Lyrer P, et al: European Stroke Organisation recommendations to establish a stroke unit and stroke center. Stroke 2013;44:828-840

12 Stroke Unit Trialists' Collaboration: Organised inpatient (stroke unit) care for stroke. Cochrane Database Syst Rev 2013;9:CD000197.

13 European Stroke Organisation (ESO) Executive Committee; ESO Writing Committee: Guidelines for management of ischaemic stroke and transient ischaemic attack 2008. Cerebrovasc Dis 2008;25:457-507.

14 Lyrer P, Michel P, Arnold M, Hungerbühler H, Gralla J, Humm A, et al: Stroke Units und Stroke Centers in der Schweiz: Richtlinien und Anforderungsprofil. Schweiz Med Forum 2012;12:918-922.

15 Sulter G, Steen C, De Keyser J: Use of the Barthel index and modified Rankin scale in acute stroke trials. Stroke 1999;30:1538-1541.

16 Van Swieten JC, Koudstaal PJ, Visser MC, Schouten HJ, van Gijn J: Interobserver agreement for the assessment of handicap in stroke patients. Stroke 1988;19:604-607.

-17 Adams HP Jr, Bendixen BH, Kappelle LJ, Biller J, Love BB, Gordon DL, et al: Classification of subtype of acute ischemic stroke. Definitions for use in a multicenter clinical trial. TOAST. Trial of Org 10172 in Acute Stroke Treatment. Stroke 1993;24:35-41.
18 Ringelstein EB, Zeumer H, Schneider R: Contribution of computer tomography of the brain to differential typology and differential therapy of ischemic cerebral infarct. Fortschr Neurol Psychiatr 1985;53:315-336.

19 Savitz SI, Lew R, Bluhmki E, Hacke W, Fisher $\mathrm{M}$ : Shift analysis versus dichotomization of the modified Rankin scale outcome scores in the NINDS and ECASS-II trials. Stroke 2007; 38:3205-3212.

20 Gattringer T, Niederkorn K, Seyfang L, Seifert-Held T, Simmet N, Ferrari J, et al: Myocardial infarction as a complication in acute stroke: results from the Austrian stroke unit registry. Cerebrovasc Dis 2014;37:147-152.

21 Silva Y, Puigdemont M, Castellanos M, Serena J, Suñer RM, García MM, et al: Semi-intensive monitoring in acute stroke and longterm outcome. Cerebrovasc Dis 2005;19:2330.

22 De Jong G, van Raak L, Kessels F, Lodder J: Stroke subtype and mortality. A follow-up study in 998 patients with a first cerebral infarct. J Clin Epidemiol 2003;56:262-268.

23 Saposnik G, Fang J, O’Donnell M, Hachinski V, Kapral MK, Hill MD: Escalating levels of access to in-hospital care and stroke mortality. Stroke 2008;39:2522-2530.

24 Di Carlo A, Lamassa M, Wellwood I, Bovis F, Baldereschi M, Nencini P, et al: Stroke unit care in clinical practice: an observational study in the Florence center of the European Registers of Stroke (EROS) Project. Eur J Neurol 2011;18:686-694. 